\title{
Fraturas supracondilianas de úmero na infância
}

\author{
Humeral supracondylar fracture in childhood
}

Carla Fumo', Roberto Rangel Bongiovanni', Eiffel Tsuyoshi Dobashi', Luciano Pascarelli', Lúcio César Silva Righi

\begin{abstract}
Resumo
Introdução: As fraturas supracondilianas do úmero são frequentes na infância e correspondem a 50 ou $60 \%$ das fraturas do cotovelo. A redução anatômica associada ou não à osteossíntese é a chave para o sucesso do tratamento independentemente do método terapêutico. Objetivo: Este trabalho teve como objetivo avaliar os resultados funcionais e radiográficos de 129 crianças cujas fraturas foram classificadas de acordo com o método de Gartland. Método: Utilizamos o protocolo prospectivo desenvolvido pela Comissão de Educação Continuada da Sociedade Brasileira de Ortopedia e Traumatologia. Resultados: Obtivemos bons resultados na avaliação final nas fraturas do tipo I e II quando aplicado o método conservador e incruento com baixos índices de complicações (3,4\%). Nas do tipo III, observamos maior número de complicações, entre elas lesões nervosas traumáticas (16,2\%) e o cúbito valgo (3,2\%). Conclusões: Para tratamento preconizamos a redução incruenta com fixação percutânea com fios de Kirschner cruzados. A redução aberta está indicada nos casos em que a redução fechada não foi bem sucedida, e a tração pré-operatória teria indicação restrita. Não preconizamos a fisioterapia para todos os pacientes, a não ser quando estes não apresentaram recuperação espontânea após um mês da retirada do gesso ou na vigência de complicação neurológica.
\end{abstract}

Palavras-chave: Fraturas do úmero; criança; terapêutica.

\section{Abstract}

Introduction: The humeral supracondylar fractures are common in childhood and correspond to 50 or $60 \%$ of the elbow fracture. Anatomical reduction associated or not with osteosynthesis is the subject for the success of treatment independent of the therapeutic method. Objective: This paper had the purpose to analyze the functional and radiographic results in 129 children whose fractures were classified according to Gartland method. Method: We applied the prospective protocol developed by the Educational Committee of the Brazilian Orthopedic and Trauma Society. Results: We observed good results at the final evaluation in fractures classified as type I and II when conservative and non-operative methods were applied with lower rate of complications $(3,4 \%)$. In type III fractures we observed higher percentage of complications as traumatic neurological damage $(16,2 \%)$ and cubitus valgus $(3,2 \%)$. Conclusions: For the treatment, we recommend closed reduction with percutaneous fixation using crossed Kirschner wires. Open reduction is indicated in cases which closed reduction was not well succeeded and pre-operative traction should has restrict indication. We do not recommend physical therapy for all patients, only in these ones that did not present spontaneous recovery after one month of the cast take-off or in the presence of neurologic complication.

Keywords: Humeral fractures; child; therapeutics.

Recebido: 8/8/2009

Revisado: 12/12/2009

Aprovado: 6/1/2010

Trabalho realizado no IFOR-Hospital do Instituto de Fraturas, Ortopedia e Reabilitação de São Bernardo do Campo, São Bernardo do Campo (SP), Brasil Hospital Instituto de Fraturas, Ortopedia e Reabilitação de São Bernardo do Campo (IFOR), São Bernardo do Campo (SP), Brasil

Endereço para correspondência: Eiffel Tsuyoshi Dobashi - Rua Américo Brasiliense, 596 - Centro - CEP 09715-021 - São Bernardo do Campo (SP), Brasil E-mail: dobashi@uol.com.br 


\section{Introdução}

As fraturas supracondilianas do úmero são frequentes na infân$\mathrm{cia}^{1-3}$ e compreendem, aproximadamente, a 50 ou $60 \%$ das fraturas do cotovelo ${ }^{2,45}$. A redução anatômica destas, associada ou não à osteossíntese estável, é a chave para o sucesso do tratamento dessa afecção, uma vez que a dificuldade em manter-se um posicionamento apropriado entre os fragmentos fraturados pode ocasionar, como resultado final, limitações do movimento articular ${ }^{1}$. Também é importante ressaltar que uma especial atenção às partes moles associada a uma criteriosa monitorização da função neuromuscular deve acompanhar a terapêutica.

Diversas metodologias têm sido utilizadas como propostas de tratamento, dentre elas: tração cutânea, tração esquelética olecraniana, redução fechada associada à imobilização gessada, redução incruenta e fixação percutânea ${ }^{1}$, redução cruenta e osteossíntese com fios de Kirschner ${ }^{1,3,6}$. Geralmente, os resultados finais, quando avaliados, são descritos como bons, independentemente do método terapêutico aplicado, embora cada um dos autores defenda sua técnica de tratamento ${ }^{1}$, apresentando sua experiência e casuística.

Os defensores do tratamento incruento baseiam-se na percepção de uma alta frequência de bons resultados, que estariam relacionados a uma compensação funcional ao nível do ombro, associada à remodelação óssea quando aplicada essa modalidade terapêutica, sendo, portanto, por muitos anos, o método de escolha de muitas escolas ortopédicas ${ }^{6}$.

Os partidários do tratamento cirúrgico indicam essa abordagem nas fraturas com desvio acentuado e advogam que a redução anatômica associada à fixação com fios de Kirschner minimiza as taxas de complicações precoces (lesão da artéria braquial, lesões dos nervos periféricos e síndrome compartimental, com possível evolução para a contratura isquêmica de Volkman ${ }^{1,4,6,7}$ ) e as tardias (miosite ossificante ${ }^{7}$, consolidação viciosa ${ }^{1,2,6-8}$, lesões fisárias ${ }^{6}$ e rigidez $\operatorname{articular}^{7}$ ).

Dessa maneira, o objetivo do estudo foi descrever os resultados clínicos, funcionais e radiográficos de pacientes portadores de fraturas supracondilianas do úmero.

\section{Método}

Para a coleta das informações foi utilizado o protocolo prospectivo de avaliação do tratamento das fraturas supracondilianas do úmero em crianças, desenvolvido pela Comissão de Educação Continuada da Sociedade Brasileira de Ortopedia e Traumatologia (SBOT) e adotado pela Sociedade Brasileira de Ortopedia Pediátrica (SBOP).

\section{Protocolo}

$1^{a}$ parte - informações pertinentes à identificação.

$2^{\mathrm{a}}$ parte - atendimento inicial:
- $\quad$ tipo de acidente, lado acometido, tipo de fratura (fechada ou exposta), mecanismo de trauma (flexão, extensão), desvio (presente ou ausente);

- classificação de Gartland" e associação com o tipo de desvio (posterolateral, anterior, posteromedial e posterior);

- perfusão capilar, pulso radial;

- lesão nervosa;

- lesões associadas (fratura do antebraço ipsilateral, do úmero proximal ipsilateral, do antebraço contralateral, do úmero contralateral e outras);

- lesões das partes moles;

- radiografias (AP, Perfil, cotovelo contralateral).

$3^{\mathrm{a}}$ parte - tratamento:

- tratamento prévio (apenas tala gessada, redução incruenta associada à tala gessada ou gesso, tração esquelética);

- tempo decorrido entre o $1^{\circ}$ atendimento até a cirurgia definitiva;

- presença de calo ósseo na época da cirurgia;

- tipo de tratamento (redução incruenta associada à fixação percutânea com fios cruzados ou paralelos, redução cruenta associada à fixação com fios cruzados);

- $\quad$ tipo acesso (lateral, medial, medial e lateral, posterior, anterior);

- complicações no pós-operatório.

$4^{a}$ parte - avaliação pós-operatória:

- controle radiográfico (ângulos de Baumann, côndilo-diafisário, carga, e úmero-ulnar);

- $\quad$ desvios (angular, rotacional, pequeno desvio, grande desvio);

- desvios na radiografia em AP (medial, lateral, ângulo);

- arco de movimento articular (flexão, extensão, pronação, supinação);

- $\quad$ seguimento (desvios, complicações).

Esta pesquisa foi aprovada pelo comitê de Ética da FMABC parecer $40 / 2009$.

\section{Resultados}

A casuística foi constituída por 129 crianças portadoras de fraturas supracondilianas do úmero (129 cotovelos), cujas idades variaram de dois a 12 anos (média de 6,2 anos). Elas foram submetidas ao tratamento (incruento ou cruento) entre janeiro de 1997 e setembro de 2001, com tempo de seguimento mínimo de 2 anos de acompanhamento (mínimo de 2 anos e máximo de 5 anos com média de 2,8 anos).

Dados referentes ao sexo das crianças, ao lado acometido, à classificação das fraturas proposta por Gartland' (tipos I, II e III) e ao local dos acidentes são apresentados na Tabela I.

Em relação ao mecanismo de lesão, extensão e flexão, nas lesões do tipo I, o trauma em extensão ocorreu em 59 (85,5\%) pacientes, con- 
Tabela I - Dados descritivos das crianças acometidas por fratura fraturas supracondilianas de úmero

\begin{tabular}{lllllllll}
\hline & \multicolumn{2}{c}{ Sexo } & \multicolumn{2}{c}{ Lado acometido } & \multicolumn{3}{c}{ Local do acidente } \\
\hline Gartland & Feminino & Masculino & Direito & Esquerdo & Domicílio & Vias públicas & Escolas & Outros \\
\hline \multirow{2}{*}{ Tipo I } & 31 & 38 & 37 & 32 & 42 & 17 & 6 & 4 \\
& $44,90 \%$ & $55,07 \%$ & $53,30 \%$ & $46,70 \%$ & $60,86 \%$ & $24,63 \%$ & $8,69 \%$ & $5,79 \%$ \\
\multirow{2}{*}{ Tipo II } & 20 & 9 & 13 & 16 & 17 & 6 & 5 & 1 \\
& $68 \%$ & $32 \%$ & $44,80 \%$ & $55,20 \%$ & $58,60 \%$ & $20,70 \%$ & $17,20 \%$ & $3,50 \%$ \\
\multirow{2}{*}{ Tipo III } & 12 & 19 & 14 & 17 & 16 & 8 & 6 & 1 \\
& $39 \%$ & $61 \%$ & $45,20 \%$ & $54,80 \%$ & $51,60 \%$ & $25,80 \%$ & $19,40 \%$ & $3,20 \%$ \\
\hline
\end{tabular}

Tabela II - Valores angulares nas radiografias em anteroposterior e Perfil

\begin{tabular}{|c|c|c|c|c|c|}
\hline Gartland & Tratamento & Baumann & $\begin{array}{l}\text { Côndilo- } \\
\text { diafisário }\end{array}$ & Carga & $\begin{array}{l}\text { Úmero- } \\
\text { ulnar }\end{array}$ \\
\hline \multirow{3}{*}{ Tipo II } & Grupo B1 & \multirow{2}{*}{$34,3^{\circ}$} & \multirow{2}{*}{$87,0^{\circ}$} & \multirow{2}{*}{$10^{\circ}$} & \multirow{2}{*}{$5,5^{\circ}$} \\
\hline & $\mathrm{n}=18(62 \%)$ & & & & \\
\hline & $\begin{array}{l}\text { Grupo B2 } \\
n=11(38 \%)\end{array}$ & $32,1^{\circ}$ & $86,3^{\circ}$ & $9,3^{\circ}$ & $5,7^{\circ}$ \\
\hline \multirow{2}{*}{ Tipo III } & $\begin{array}{l}\text { Grupo C1 } \\
n=20(64,5 \%)\end{array}$ & $30,1^{\circ}$ & $84,2^{\circ}$ & $9,2^{\circ}$ & $5,0^{\circ}$ \\
\hline & $\begin{array}{l}\text { Grupo C2 } \\
n=11(34,5 \%)\end{array}$ & $33,4^{\circ}$ & $80^{\circ}$ & $9,3^{\circ}$ & $5,6^{\circ}$ \\
\hline
\end{tabular}

Tabela III - Arco de movimento pós-tratamento

\begin{tabular}{llccc}
\hline Gartland & Flexão & Extensão & Pronação & Supinação \\
\hline Tipo I & $131,20^{\circ}$ & $2,3^{\circ}$ & $79,1^{\circ}$ & $78^{\circ}$ \\
Tipo II & $139,2^{\circ}$ & $4,3^{\circ}$ & $81,2^{\circ}$ & $73,1^{\circ}$ \\
Tipo III & $135^{\circ}$ & $4,0^{\circ}$ & $80^{\circ}$ & $75^{\circ}$ \\
\hline
\end{tabular}

tra $10(14,5 \%)$ em flexão. No tipo II, o mecanismo em extensão foi encontrado em 24 (83\%) situações e a flexão em 5 (17\%). Por fim, no tipo III, o trauma em extensão ocorreu em $26(83,9 \%)$ lesões e em flexão, em 5 (16,1\%). Nenhuma das crianças apresentou lesões da pele ou exposição óssea.

Com relação ao desvio das fraturas, no tipo II, o desvio principal foi anterior em 22 (75,8\%) pacientes e o desvio posterior acometeu 7 (25,2\%). Já no tipo III, foram $16(51,6 \%)$ para anterior e $15(48,4 \%)$ para posterior.

A Tabela II apresenta os valores angulares encontrados nas radiografias nas incidências anteroposterior (AP) e perfil, nas lesões de tipo II e III.

Em relação ao tratamento, as fraturas do tipo I de Gartland ${ }^{9}$ foram tratadas pelo método conservador utilizando-se imobilização com aparelho gessado axilo-palmar em posição neutra por um período de, aproximadamente, três semanas. Não foram observadas complicações ou limitações funcionais, conforme arco de movimento médio apresentado (Tabela III).

No grupo em que as fraturas foram classificadas como tipo II de Gartland ${ }^{9}$, nenhuma criança apresentou lesões cutâneas importantes e o edema foi considerado moderado. Cinco (17\%) crianças com fraturas com desvio anterior e instabilidade foram submetidas à redução fechada e utilizada osteossíntese com dois fios de Kirschner cruzados, 10 (35\%) foram submetidas à redução incruenta e 14 (48\%) apenas à imobilização com tala gessada axilo-palmar por 3 ou 4 semanas.

O tratamento definitivo imediato foi realizado em 18 (62\%) pacientes, grupo B1 (Tabela II), e após 12 horas em 11 (38\%), grupo B2 (Tabela II). Quanto ao tratamento fisioterapêutico $6(20,7 \%)$ crianças foram submetidas à terapêutica por 5 semanas em média. $O$ cúbito varo ocorreu em 1 (3,4\%) paciente, para o qual foi proposto tratamento conservador, e foi percebido após um ano de seguimento.

Dos pacientes com fraturas do tipo III de Gartland', todos apresentaram perfusão normal, mas o pulso radial apresentou-se diminuído em quatro $(12,9 \%)$ deles. $\mathrm{Na}$ avaliação primária foram encontradas apenas uma $(3,2 \%)$ neuropraxia do nervo radial e duas (6,5\%) fraturas do antebraço ipsilateral associadas ao trauma em extensão. Quanto à lesão dos tecidos moles, as escoriações foram percebidas em $5(16,1 \%)$ pacientes e o edema do membro superior acometido era substancialmente maior.

$\mathrm{O}$ tratamento seguiu duas linhas terapêuticas específicas. No primeiro grupo, grupo C1 (Tabela II), 20 (64,5\%) pacientes foram submetidos ao tratamento operatório imediato, ou seja, foram internados com menos de quatro horas desde a ocorrência da fratura e submetidos à anestesia geral e reduções de suas fraturas, associadas à osteossíntese com dois fios de Kirschner cruzados. Destes, 16 (51,61\%) foram submetidos à redução incruenta e $4(12,9 \%)$ à redução aberta por via posterior.

O grupo C2 (Tabela II) foi constituído por 11 pacientes (34,5\%) submetidos inicialmente à tração esquelética olecraniana em virtude do grande edema do membro superior acometido e do período superior a 8 horas desde a ocorrência das lesões. Após 2 ou 5 dias, os pacientes foram submetidos ao tratamento definitivo de suas fraturas, sendo que em 7 (22,58\%) deles optou-se pela redução fechada associada à fixação com dois fios de Kirschner cruzados e em 4 (12,9\%), pela redução aberta por via posterior e fixação com dois fios de Kirschner cruzados.

$\mathrm{Na}$ avaliação pós-operatória desses pacientes, observou-se lesão do nervo ulnar e nervo radial em 1 (3,2\%) paciente que foi submetido ao tratamento imediato e $1(3,2 \%)$ paciente com lesão do nervo ulnar apenas. Outros 2 (6,5\%) apresentavam lesão do nervo ulnar, quando foram tratados primeiramente pela tração esquelética olecraniana. Nenhum deles apresentava sinais de lesão nervosa no exame pré-operatório e optou-se pelo tratamento de redução fechada, associado à osteossínte- 
se. Quatro (12,9\%) dentre os 11 pacientes apresentaram lesões nervosas e foram submetidos à neurorrafia após dois meses de tratamento.

Nas radiografias em AP do período pós-operatório imediato foram visualizados pequenos desvios em 4 (12,9\%) pacientes, sendo 3 $(9,7 \%)$ medial e $1(3,2 \%)$ lateral, quando foram tratados por método incruento. Os valores dos arcos de movimento desse grupo estão na Tabela III.

Do total de 31 pacientes, 17 (54,83\%) foram encaminhados ao serviço de fisioterapia, e 5 (16,1\%) apresentavam complicações pré e pós-operatórias (lesões nervosas) e foram acompanhados por um período médio de 10 semanas, quando tiveram suas funções neuromotoras restabelecidas.

\section{Discussão}

Existem, basicamente, três fatores que predispõem a região supracondiliana do úmero às fraturas na infância e na adolescência: menor diâmetro ósseo do local, o que diminui a resistência mecânica do úmero; afilamento da fossa olecraniana; e frouxidão ligamentar. A combinação desses fatos deve, de certa forma, explicar a alta frequência desse tipo de lesão óssea especialmente na população pediátrica.

Quanto ao mecanismo de trauma, a reação protetora reflexa dos indivíduos submetidos ao acidente, nos quais os membros superiores são posicionados estendidos, determinam, portanto, uma hiperextensão do cotovelo, o que se observa na maioria dos $\operatorname{casos}^{7}$ concordando com nossa casuística (82,9\%), sendo o ambiente domiciliar o mais comum.

Com relação ao tratamento, essas fraturas são consideradas urgências médicas ${ }^{3,7}$ e o objetivo principal é restituir totalmente a função e a anatomia local evitando-se, com isso, as complicações ${ }^{2}$. O tratamento definitivo, a ser aplicado por um cirurgião experiente, deve ser o mais eficaz e rápido possível ${ }^{6,7}$ segundo os autores da literatura.

Nem sempre, entretanto, acontece uma intervenção imediata ${ }^{7} \mathrm{em}$ razão da ausência de profissionais especializados, das condições cirúrgicas inadequadas e da falta de material cirúrgico apropriado; fatores esses imprescindíveis para que seja feita uma projeção favorável de bons resultados após a instituição da terapia ${ }^{7}$. Muitas vezes, podemos até aguardar um determinado período de tempo, especialmente quando é possível completar-se o jejum pré-operatório, desde que o paciente não apresente exposição do foco de fratura ou não sejam percebidos sinais da isquemia do membro acometido ${ }^{6}$. Carmichael e Joyner ${ }^{10}$, Walmsley et al. ${ }^{11}$ e Sibinski et al. ${ }^{12}$ afirmam que, sendo observados esses dois princípios, não há alteração significativa no resultado quando se aguarda tempo para regressão do edema e condições cirúrgicas apropriadas (material e equipe), além de não interferir, nos casos do tipo III de Gartland ${ }^{9}$, na decisão entre redução cruenta ou incruenta ${ }^{11}$, como observamos em nossa casuística.

Independentemente da modalidade de tratamento escolhida é de consenso que há importância em se obter uma redução exata entre os fragmentos fraturados ${ }^{1,2}$, tomando cuidado especial com os des- vios rotacionais que seriam determinantes para o aparecimento de deformidades, principalmente nas fases tardias ${ }^{8,9}$. Ainda assim, existem defensores que afirmam que uma redução pouco anatômica não acarreta, obrigatoriamente, um dano estético ou funcional, visto que o crescimento e a remodelação óssea podem contribuir sobremaneira para corrigir eventuais desvios ${ }^{3}$

Nas fraturas com desvio, a redução fechada associada à aplicação do aparelho gessado tem sido relacionada com a maior incidência da isquemia de Volkman e do desvio em varo ${ }^{1}$. Quando uma flexão maior que $90^{\circ}$ é requerida para manter a redução, são necessários cuidados extremos e julgamento apropriado, pois, nessa posição, a circulação do membro superior pode ser comprometida ${ }^{1,7}$. Contudo, quando um grau menor de flexão é aplicado, há grande chance de perda da posição entre os fragmentos da fratura'.

A contratura isquêmica é a mais grave complicação das fraturas nas crianças e, no membro superior, a fratura supracondiliana do úmero é a principal causadora, segundo Seddon ${ }^{4}$. Sua incidência vem decaindo ao longo das últimas décadas, provavelmente pela decorrência de um melhor conhecimento de sua fisiopatologia ${ }^{4}$ e, muito provavelmente, pela precocidade e precisão na sua identificação, pela aplicação de medidas clínicas essenciais (observação constante do pulso radial e perfusão da mão), pela avaliação atenta das queixas da criança, especialmente dor, e pela aplicação do tratamento definitivo apropriado das fraturas. Não constatamos essa alteração. Entretanto, no grupo em que as fraturas foram classificadas como tipo III de Gartland", salientamos que há iminente preocupação, visto que clinicamente foram constatados os maiores edemas do membro superior e um déficit do pulso radial (12,9\%) nesse grupo.

Um método adjuvante ao tratamento é a utilização da tração cutânea ou esquelética que ainda encontra seus defensores ${ }^{1}$. Essa técnica, além de não trazer alteração significativa em nossa casuística, é acompanhada de uma série de desvantagens, entre elas relacionamos: o período prolongado de internação antes de iniciar-se o tratamento definitivo; exposição ao risco anestésico (para a aplicação da tração e, muitas vezes, uma segunda para executar o tratamento definitivo); dificuldades na sua manutenção; maior incidência de cúbito varo, 33\% no estudo de Piggot ${ }^{13}$, maior risco de lesão neurológica, vascular e fisária; fratura do olecrano.

Sua aplicação estaria restrita, após minuciosa análise, a situações especiais como: falta de condições para aplicação do tratamento definitivo, percepção de um volumoso edema local e presença de extensas lesões cutâneas. Isso ocorreu em 11 (34,5\%) pacientes com fraturas do tipo III quando a redução e fixação foram realizadas num segundo tempo, sem diferença estatística entre os dois grupos $(p>0,05)$.

A fixação percutânea é extremamente popular ${ }^{1-4,6}$. Foi inicialmente descrita por Judet e Swenson como técnica de osteossíntese, baseados no trabalho de Miller (1939) que indica essa metodologia para o tratamento das lesões em forma de " $T$ ”". Seus partidários alegam que a sua ampla utilização se deve a uma série de vantagens como o seu baixo custo, a segurança e eficiência do método e a permanência hospitalar mínima ${ }^{1}$. 
A utilização de um único fio lateral como opção de tratamento demonstra ser altamente eficaz ( $80 \%$ de resultados satisfatórios), entretanto quando aplicada, são comuns os desvios secundários como complicação $0^{14}$. O uso de dois fios laterais torna desnecessária a associação de uma flexão aguda do cotovelo para a manutenção da redução. Além do mais, seus defensores alegam que esse método teria como principal vantagem minimizar os riscos da ocorrência da lesão do nervo ulnar, pois não seria necessária a passagem do fio medial ${ }^{2}$.

O método de osteossíntese que emprega dois fios cruzados para a fixação das fraturas determina altos índices de resultados satisfatórios $(98 \%)$ como consequência de grande estabilidade, simplicidade de aplicação, curto período de internação hospitalar, manutenção da anatomia restaurada e diminuição dos riscos do aparecimento da síndrome compartimental ${ }^{14}$. Essa estabilização é decorrente de um efeito biomecânico, no qual é aproveitada a força aplicada no sentido medial dada pela deformação elástica do fio denominado "efeito mola",14. Alguns autores contestam esse tipo de fixação temendo que ocorra um desvio rotacional do fragmento distal quando um dos fios é anteriormente posicionado ao outro ${ }^{6}$. A osteossíntese de todas as fraturas contidas em nosso trabalho seguiu esse princípio e concluímos que a utilização de aparelho de radioscopia facilita em muito o emprego dessa técnica, pois permite que a passagem dos fios de Kirschner seja efetuada num menor período de tempo possível. Preconizamos que uma mínima incisão medial seja realizada para que o epicôndilo medial possa ser visualizado, minimizando os riscos de lesão iatrogênica do nervo ulnar.

A opção em deixar os fios expostos transcutaneamente ${ }^{14}$ ou em mantê-los contidos logo abaixo da pele depende da preferência de cada cirurgião. A metodologia de estabilização percutânea, apesar de ser eficaz, não está livre de complicações porque sempre há risco iminente de infecção pelo trajeto dos fios. A migração também pode ocorrer ${ }^{14}$ e, quando notada, coloca em risco a redução obtida, principalmente nas fraturas instáveis com desvio. Não observamos, contudo, tais complicações em nossa casuística. Nossos pacientes foram seguidos semanalmente quando submetidos ao curativo de suas feridas operatórias, sendo, então, mantidos com imobilização gessada.

Quando é necessária uma abordagem aberta das fraturas, a via de acesso posterior com subsequente isolamento do nervo ulnar permite acesso ao músculo tríceps, medial e lateral, sem realizar sua desinserção ou secção transversal, método preconizado para evitar retrações cicatriciais e limitação funcional ${ }^{3}$. Essa via cirúrgica foi utilizada para o tratamento das fraturas tipo III de Gartland seguindo os mesmos princípios relatados anteriormente.

A via de acesso anterior é defendida por outros autores ${ }^{5}$ devido à necessidade de menor dissecção cirúrgica para abordar a fratura, visto que grande parte do músculo braquial se encontra danificado, principalmente nas lesões mais graves; é possível, por essa via, reconhecer, isolar e proteger o feixe vásculo-nervoso durante a manobra de redução; ela também permite redução anatômica por visão direta e facilita o controle da fratura durante o período pós-operatório.
Kaewpornsawan ${ }^{15}$, em ensaio clínico randomizado, não encontrou diferenças nos resultados do tratamento cirúrgico entre o método cruento e incruento, no entanto ressalta que o método percutâneo seja a primeira tentativa numa fratura fechada.

É importante ressaltar que, muitas vezes, a pesquisa clínica dos sinais de lesão neurológica não é tarefa fácil, pois a criança, devido à dor, não colabora suficientemente quando realizado o exame sensorial e motor ${ }^{6}$. Acreditamos, contudo, que uma avaliação criteriosa pré e pósoperatória deva ser realizada sempre.

As lesões nervosas, como as neuropraxias, são relativamente frequentes e seus índices variam de 6 a 16\% das complicações ${ }^{14,16}$ e ocorrem tanto pelo trauma como pela iatrogenia (2,79 a 6,25\%), em decorrência da passagem dos fios de Kirschner para a realização da osteossíntese ou de uma manipulação intempestiva ${ }^{7}$. Em muitas situações, o grande edema da região torna difícil o reconhecimento adequado dos acidentes anatômicos que devem ser palpados para que uma precisa fixação possa ser aplicada ${ }^{3}$ Logo, não raramente, a lesão transfixante do nervo ulnar decorre pela fixação ${ }^{3}$.

A afirmação de que o nervo radial é o mais lesado nestas circunstâncias ${ }^{4,16}$ não é unânime. $O$ interósseo anterior é frequentemente reconhecido como o mais acometido quando associado às fraturas supracondilares do úmero ${ }^{16}$. O mecanismo de lesão se deve à tração longitudinal dessa estrutura ou ao trauma direto de sua porção anterior junto a uma das extremidades da fratura ${ }^{16}$. Por exemplo, citamos o trabalho de Volpi et al. ${ }^{1}$, que observam dois pacientes acometidos por lesões temporárias do nervo ulnar. Em nossa casuística, observamos quatro lesões nervosas $(12,9 \%)$ do ulnar associadas ao tipo III de Gartland' que foram tratadas pela neurorrafia e uma lesão do nervo radial $(3,2 \%)$.

Tardiamente, a neurite do nervo ulnar é citada como causadora de dor no nível do cotovelo. É secundária ao aparecimento do cúbito valgo e requer, como tratamento, a transposição anterior do nervo ulnar?. Não observamos essa complicação.

A transfixação da fise de crescimento pelos fios lisos não parece determinar lesões da placa de crescimento, pois seu diâmetro não seria suficientemente calibroso para determinar esse tipo de lesão e a permanência por pequenos períodos de tempo não interferiria na sua conformação histológica.

O cúbito varo ou valgo, como intercorrência tardia, teria relação com reduções imperfeitas, visto que essa problemática não é acompanhada pelas lesões da cartilagem de crescimento no nível do cotovelo, as quais provocariam a deformidade ${ }^{14,16}$. A rotação interna do fragmento distal também é considerada na gênese dessa deformidade ${ }^{8}$. A lesão da fise de crescimento é, segundo alguns autores, a causa do cúbito varo, que varia de 7 a $57 \%$, pela ocorrência de um distúrbio do local do crescimento, porém existem aqueles que acreditam numa persistência da inclinação medial do fragmento distal como sendo o motivo mais importante ${ }^{2}$. Observamos essa complicação em um $(3,2 \%)$ paciente com fratura do tipo II de Gartland ${ }^{9}$ provavelmente pela lesão da fise associada a uma redução imperfeita quando fizemos uma análise retrospectiva do caso. 
A avaliação radiográfica pré-operatória deve ser vista com bastante critério em virtude da grande proporção de estruturas cartilaginosas, principalmente nas crianças muito jovens. Como critérios de avaliação pós-operatória são considerados diversos parâmetros angulares e sua análise auxilia na qualificação dos resultados ${ }^{17}$. O ângulo de carregamento determina bons resultados quando menor que $10^{\circ 5}$. Os parâmetros radiográficos por nós estudados não evidenciaram desvios da normalidade quando os correlacionamos com os diferentes tipos de Gartland'. Observamos que, na radiografia pós-operatória em perfil, quando a projeção da cortical anterior do úmero não cruzava o côndilo lateral dividindo-o em $2 / 3$ anteriores e $1 / 3$ anterior, havia perda da extensão do cotovelo.

Na literatura ortopédica são poucos os relatos a respeito do processo de reabilitação nesses casos ${ }^{7}$. A maioria compartilha a opinião de que um retorno espontâneo da capacidade funcional é obtido sem a necessidade de intervenção fisioterapêutica. A reabilitação geralmente é requerida quando é percebido um prejuízo das atividades de vida diária ao final do primeiro mês após a retirada da imobilização?

São observadas limitações da amplitude do movimento articular com maior frequência quando é feita a opção pela redução e fixação cruenta $^{7}$. Quando é utilizada a via de acesso posterior, são comuns os relatos de miosite ossificante, cicatriz hipertrófica e, principalmente, limitação funcional. Tal fato pode estar relacionado com a formação de tecido fibroso cicatricial junto à fossa olecraniana e aos períodos prolongados de imobilização?

Com atenção especial à miosite ossificante, esta pode também ser decorrente da mobilização passiva forçada na tentativa de ganhar a extensão ${ }^{7}$. Não acreditamos que um período maior de imobilização possa permitir regressão dessa entidade nosológica, como observamos na literatura?

A hipotrofia pelo desuso, associada à perda funcional e potencial, não afeta somente os diferentes grupos musculares, mas também compromete o tecido ósseo de todo o membro superior afetado 7 . É preconizado que atividades lúdicas, cujo objetivo é tornar a terapia eficaz e prazerosa para a criança, devam ser prescritas assim como a hidrotera- pia, pois o alívio da dor e a diminuição do espasmo muscular podem ser rapidamente alcançados?

O período em que pode ocorrer a recuperação da mobilidade articular é variável, contudo espera-se que ocorra de modo total entre o primeiro e quarto ano após a fratura ${ }^{2}$. Na reabilitação das neuropraxias, o retorno espontâneo da função é esperado entre a $\sigma^{\mathrm{a}} \mathrm{e} 16^{\mathrm{a}}$ semana ${ }^{7,16}$. Keppler et al. ${ }^{18}$ mostram, em seu trabalho, que a fisioterapia é desnecessária nas fraturas supracondilianas de úmero, tendo papel reservado nas que possuem comprometimento neurológico para a estimulação acelerar a recuperação motora e sensitiva. Submetemos $5(16,2 \%)$ de nossos pacientes portadores de lesão neurológica ao tratamento fisioterápico, os quais recuperaram suas funções em aproximadamente 10 semanas de tratamento quando foi instituída a termoterapia, a cinesioterapia e a estimulação elétrica.

Nas fraturas do tipo I de Gartland', o método preconizado deve ser conservador com imobilização gessada por três ou quatro semanas. Não foram observadas complicações. Nas do tipo II, a redução incruenta simples ou, eventualmente, a redução incruenta associada à fixação percutânea com fios de Kirschner cruzados estão indicados e o resultado final tende a ser bom. Observamos, nas fraturas do tipo III, maiores índices de complicações, concordando com as informações da literatura ortopédica ${ }^{3,6,9}$ e os resultados finais foram semelhantes ${ }^{9}{ }^{19}$. Não foi indicada a tração cutânea ou esquelética como método de rotina, devendo ser preconizada somente em situações especiais.

Considera-se que a tentativa de redução incruenta das fraturas mais graves deve ser única. O insucesso ante essa proposição de tratamento deve imediatamente ser seguido pelo acesso direto da fratura pela via cirúrgica mais apropriada, seja anterior ou posterior.

Assim, a redução incruenta ou cruenta associada à fixação percutânea apresenta altos índices de resultados clínicos e radiográficos satisfatórios. Quanto mais grave a fratura, de acordo com os critérios de Gartland, maior a frequência de complicações, como, por exemplo, as lesões nervosas traumáticas ou iatrogênicas do nervo ulnar. Não preconizamos a fisioterapia para todos os pacientes, a não ser quando não apresentam recuperação espontânea após um mês da retirada do gesso ou na vigência de complicação neurológica.

\section{Referências}

1. Volpi MS, Jordan AM, Lepipico NC, Nogueira AC, Pereira HR. Redução fechada e fixação percutânea em fraturas supracondilianas do úmero em crianças. Rev Bras Ortop. 1997;32(12):975-81.

2. Bertol P, Monteggia GM, Paula MD. Fixação percutânea das fraturassupracondilianas do úmero na criança. Rev Bras Ortop. $1991 ; 26(3): 48-51$

3. Hungria Neto JS, Fregoneze M, Mercadante MT, Teixeira AAA, Yoneda T. Fraturas supracondilianas do úmero em crianças: avaliação do resultado do tratamento cirúrgico. Rev Bras Ortop. 1996;31 (7): 575-82.
4. Hespanhol CB, Vieira RLC, Mattuella F, Paciornik IL, Hespanhol WB, Castro AAR. Fratura supracondiliana do úmero na criança: redução incruenta com fixação percutânea. Acta Ortop Bras. 1997;5(3):110-4.

5. Sobania LC, Sanches CL, Vons DK. Fratura supracondiliana umeral em criança, estudo e avaliação de 183 casos; Especial referência ao tratamento cirúrgico. Rev Bras Ortop. 1985;20(3):89-95.

6. Amatuzzi MM, Zoppi Filho A, Montenegro NB. Fratura supracondiliana do úmero em crianças: estudo de 90 casos operados. Rev Bras Ortop. 1997;32(6):437-42. 
7. Brigidio PAF, Faria TCC, Sposito MMM, Masiero D. Fratura supracondiliana do úmero em crianças: conduta fisioterapêuta baseada em atividades lúdicas. Med Reabil. 2001;(56):25-8.

8. French PR. Varus deformity of the elbow following supracondylar fractures of the humerus in children. Lancet. 1959;2(7100):439-41.

9. Gartland JJ. Management of supracondylar fractures of the humerus in children. Surg Gynecol Obstet. 1959;109(2):145-54.

10. Carmichael KD, Joyner K. Quality of reduction versus timing of surgical intervention for pediatric supracondylar humerus fractures. Orthopedics. 2006;29(7):628-32.

11. Walmsley PJ, Kelly MB, Robb JE, Annan IH, Porter DE. Delay increases the need for open reduction of type-III supracondylar fractures of the humerus. J Bone Joint Surg Br. 2006;88(4):528-30.

12. Sibinski M, Sharma H, Bennet GC. Early versus delayed treatment of extension type-3 supracondylar fractures of the humerus in children. J Bone Joint Surg Br. 2006;88(3):380-1.

13. Piggot J, Graham HK, McCoy GF. Supracondylar fractures of the humerus in children treatment by straight lateral traction. J Bone Joint Surg Br. 1986;68(4):577-83
14. Croci AT, lacovone M, Silveira CG, Oliveira Jr EJ. Fixação percutânea de efeito dinâmico-elástico nas fraturas supracondilianas do úmero em crianças: estudo de 18 casos. Rev Bras Ortop. 1992;27(1/2):71-4.

15. Kaewpornsawan K. Comparison between closed reduction with percutaneous pinning and open reduction with pinning in children with closed totally displaced supracondylar humeral fractures: a randomized controlled trial. J Pediatr Orthop B. 2001;10(2):131-7.

16. Dormans JP, Squillante R, Sharf $H$. Acute neurovascular complications with supracondylar humerus fracture in children. J Hand Surg A. 1995:20(1):1-4

17. Canto FW, Silva DB, Santos HF, González LE, Velasco PA, Karam FC et al. Fraturas supracondilianas do úmero em crianças: análise estatística e resultados radiológicos pós-consolidação imediata por fixação percutânea com fios de Kirschner. Acta Méd. 1994;15:47-54.

18. Keppler P, Salem K, Schwarting B, Kinzl L. The effectiveness of physiotherapy after operative treatment of supracondylar humeral fractures in children. $J$ Pediatr Orthop. 2005;25(3):314-6

19. Martini RK, Fonseca GF, Martini RK, Azevedo Filho M, Serafini OA. Análise das fraturas supracondilianas do úmero em crianças. Acta Ortop Bras. 2002;10(2):25-30 